\title{
INDEX for Volume 93
}

B. Ammann, M. Dahl, \& E. Humbert, Smooth Yamabe invariant

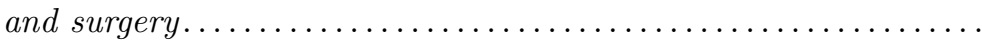

J. An, J.-K. Yu, \& J. Yu, On the dimension datum of a subgroup and its application to isospectral manifolds.................

V. Bangert \& P. Emmerich, Area growth and rigidity of surfaces

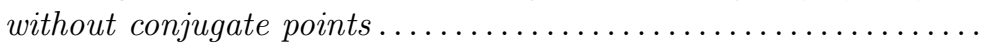

M. Bauer, P. Harms \&P.W. Michor, Sobolev metrics on the manifold of all Riemannian metrics .......................

S. Brendle \& M. Eichmair, Isoperimetric and Weingarten surfaces

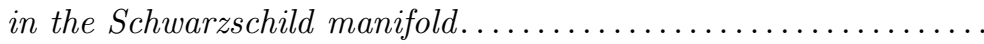

A. Chau \& L.-F. Tam, Kähler $C$-spaces and quadratic bisectional

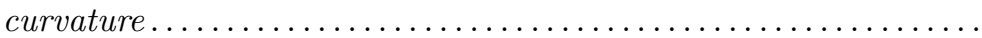

Z. Chen, M. Stiénon, \& P. Xu, Poisson 2-groups..............

Q.-S. Chi, Isoparametric hypersurfaces with four principal curva-

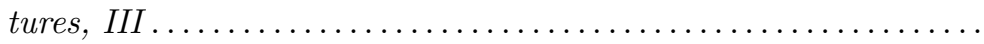

D. DeTurck \& H. Gluck, Linking, twisting, writhing, and helicity

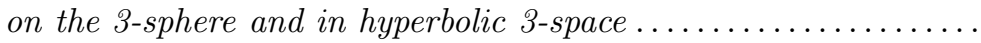

M. Eastwood \& H. Goldschmidt, Zero-energy fields on complex

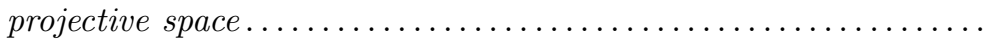

M Eichmair \& J. Metzger, Large isoperimetric surfaces in initial data sets .......................................

J. Head, On the mean curvature evolution of two-convex hyper-

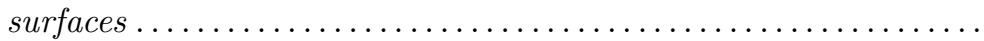

M. Mirzakhani, Growth of Weil-Petersson volumes and random hyperbolic surfaces of large genus .....................

T. Okuda, Classification of semisimple symmetric spaces with

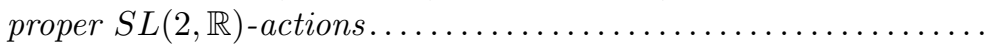

R. C. Penner, Tropical lambda lengths, measured laminations and

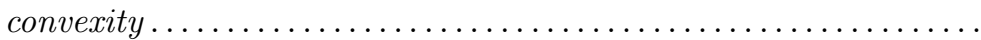

J. Seiler \& A. Strohmaier, On the noncommutative residue for

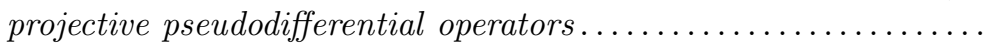

Z. Tang \& W. Yan, Isoparametric foliation and Yau conjecture on the first eigenvalue... 